\title{
Certezas e incertidumbres en la etapa posdoctoral de la carrera científica en la Argentina
}

\author{
M. Fernanda Reyes ${ }^{1,2,}$, ; M. Victoria Piazza ${ }^{1,2,3}$; Marta C. Telesnicki ${ }^{12,4}$; Luciana \\ D’Acunto ${ }^{1,2}$; Carla E. Di Bella ${ }^{1,2,5}$; Florencia Spirito ${ }^{1,2}$; Pablo A. García-Parisi ${ }^{5,6}$; \\ Josefina L. De Paepe ${ }^{1,7}$ \& Romina A. Cavagnaro ${ }^{1,8}$

\begin{abstract}
${ }^{1}$ Universidad de Buenos Aires. Facultad de Agronomía. Buenos Aires, Argentina (FAUBA). ${ }^{2}$ Consejo Nacional de Investigaciones Científicas y Técnicas (CONICET). Instituto de Investigaciones Fisiológicas y Ecológicas Vinculadas a la Agricultura (IFEVA). ${ }^{3}$ Departamento de Producción Vegetal. Cátedra de Dasonomía, FAUBA. ${ }^{4}$ Departamento de Métodos ${ }^{8}$ Departamento de Recursos Naturales y Ambiente. Cátedra de Botánica Sistemática, FAUBA.
\end{abstract} \\ Cuantitativos y Sistemas de Información, FAUBA. ${ }^{5}$ Departamento de Producción Animal. Cátedra de Forrajicultura, FAUBA. \\ ${ }^{6}$ Centro de Investigaciones y Transferencia del Noroeste de la Provincia de Buenos Aires (CITNOBA, CONICET-UNNOBA), \\ Pergamino. ${ }^{7}$ Departamento de Ingeniería Agrícola y Uso de la Tierra. Cátedra de Fertilidad y fertilizantes, FAUBA-CONICET.
}

\begin{abstract}
Resumen. En la etapa posdoctoral varios desafíos e interrogantes determinan nuestra consolidación como investigadores. Sobre la base de reuniones con ocho investigadores formados, identificamos algunas certezas e incertidumbres de esta etapa. También destacamos herramientas para lograr buenos antecedentes académicos y poder acceder a la Carrera de Investigador Científico (CIC) del Consejo Nacional de Investigaciones Científicas y Técnicas (CONICET). La principal certeza fue que hay que publicar: es fundamental para la trasmisión del conocimiento a la comunidad y es el aspecto más valorado por el CONICET. Sin embargo, publicar implica mucho más que redactar un manuscrito. Es fundamental la contribución conceptual y la originalidad de nuestro trabajo. Identificamos la incertidumbre que nos genera cómo administrar el tiempo para lograr varias publicaciones de alto impacto y realizar otras actividades como formar recursos humanos, hacer docencia o pedir subsidios o estadías en el exterior, durante los siete años de becas que normalmente disponemos (cinco para doctorarnos y dos de posdoctorado). Otras incertidumbres son el cambio constante y la exigencia creciente de requisitos para ingresar a la CIC. Además, la poca estabilidad de los recursos para investigar y la necesidad de buscar ideas originales son incertidumbres importantes en esta etapa. Quienes quieran desarrollar una carrera científica en la Argentina tendrán que delinear una estrategia. Proponemos perfiles de becarios posdoctorales 'exitosos' a fin de encontrar alternativas eficientes para acceder a una posición y un buen desarrollo de la carrera de científico en el CONICET. Para generar un debate enriquecedor, valoraremos su opinión en una encuesta que diseñamos especialmente (goo.gl/forms/k185nQG8ABxjL4bo2, abierta hasta 31 de marzo de 2019). La encuesta está dirigida a becarios doctorales, posdoctorales e investigadores con un cargo permanente, y nos permitirá abrir el abanico de perfiles y opiniones. Este debate servirá de guía a jóvenes que quieran desempeñarse exitosamente como científicos en nuestro país.
\end{abstract}

[Palabras clave: balance de actividades, interrogantes, investigadores en formación, publicaciones científicas, vocación]

\begin{abstract}
Aвstract. Certainties and uncertainties affecting the postdoctoral phase of the scientific career in Argentina. In the postdoctoral stage several challenges and questions define our development and consolidation as researchers. Based on the guidance of trained researchers, we identified various certainties and uncertainties during this phase. We also highlight several tools to achieve a good academic performance and obtain a position as researchers of the Consejo Nacional de Investigaciones Científicas y Técnicas (CONICET). The main certainty was that publishing is a 'must': it is essential to transmit the knowledge to the community and it is the most valued aspect by the CONICET. However, a publication involves much more than writing a manuscript. Detecting the conceptual contribution and the originality of our work is fundamental. One of the uncertainties identified was producing several high-impact publications, while performing other activities (e.g., human resources training, teaching, grant applications and stays abroad) during the seven years that fellowships usually last (five for Ph.D. and two for postdoctoral fellowships). Other uncertainties are the constantly changing rules and the increasing requisites to meet CONICET conditions for researchers. Besides, the scarce stability of resources to research and the need to develop original ideas are particularly uncertain at this stage. Those wanting to develop a scientific career in Argentina must outline a strategy. We propose 'successful' postdoctoral fellows' profiles to find pathways towards a permanent position at the CONICET scientific career, with good development prospects. As a way to open a fruitful debate, your survey feedback will be highly valued. Said survey is available at (goo.gl/forms/k185nQG8ABxjL4bo2, until March 31't, 2019), addressed to doctoral students, postdoctoral fellows and researchers with permanent positions. This will allow opening the range of profiles and opinions. This debate may be a guide for those young researchers willing to perform successfully as Scientists within our country.
\end{abstract}

[Keywords: training researchers, scientific publications, activities balance, question marks]

Editor asociado: Esteban Jobbágy
Recibido: 2 de abril de 2018

Aceptado: 29 de junio de 2018 


\section{INTRODUCCIÓN}

En los institutos y universidades trabajan becarios, técnicos e investigadores de planta permanente, entre otros. Los becarios son jóvenes profesionales que obtienen una beca para realizar su formación académica, primero en el marco de carreras de maestría o doctorado. Luego de la defensa del doctorado existen becas posdoctorales cuyo propósito es continuar la formación como investigadores y construir una línea de investigación. Al finalizar esta etapa de formación, quienes pretendan continuar en ciencia deberán postularse para obtener una posición permanente en su lugar de trabajo. El acceso a una posición de mayor estabilidad hace posible consolidarse como investigadores formados y generar una línea de investigación propia. Con el término carrera científica nos referiremos específicamente a una de las alternativas de desarrollo de la ciencia en la Argentina, que es la Carrera de Investigador Científico (CIC) del Consejo Nacional de Investigaciones Científicas y Técnicas (CONICET). Para lograr acceder a la CIC es necesario presentarse a la convocatoria que se realiza todos los años y en la que se evalúan de manera exhaustiva los antecedentes de los candidatos. Por lo tanto, en las etapas de formación doctoral y posdoctoral es cuando debemos consolidar los requisitos necesarios para lograr el ingreso a la CIC.

Este debate se inició cuando todos los autores éramos becarios posdoctorales de la Facultad de Agronomía de la UBA e, impulsados por las incertidumbres asociadas a nuestra formación como investigadores, generamos un espacio de discusión. Nuestros interrogantes comunes pasaban por cómo ser exitosos en cuestiones académicas específicas de nuestro quehacer y también por repensar nuestra vocación como investigadores. En la búsqueda de respuestas y consejos nos reunimos con ocho investigadores formados del CONICET, vinculados con nuestros lugares de trabajo, pero con distintos años de experiencia y diversas líneas de investigación (dentro del área de las ciencias agrarias). Les pedimos que compartieran de manera informal los aspectos

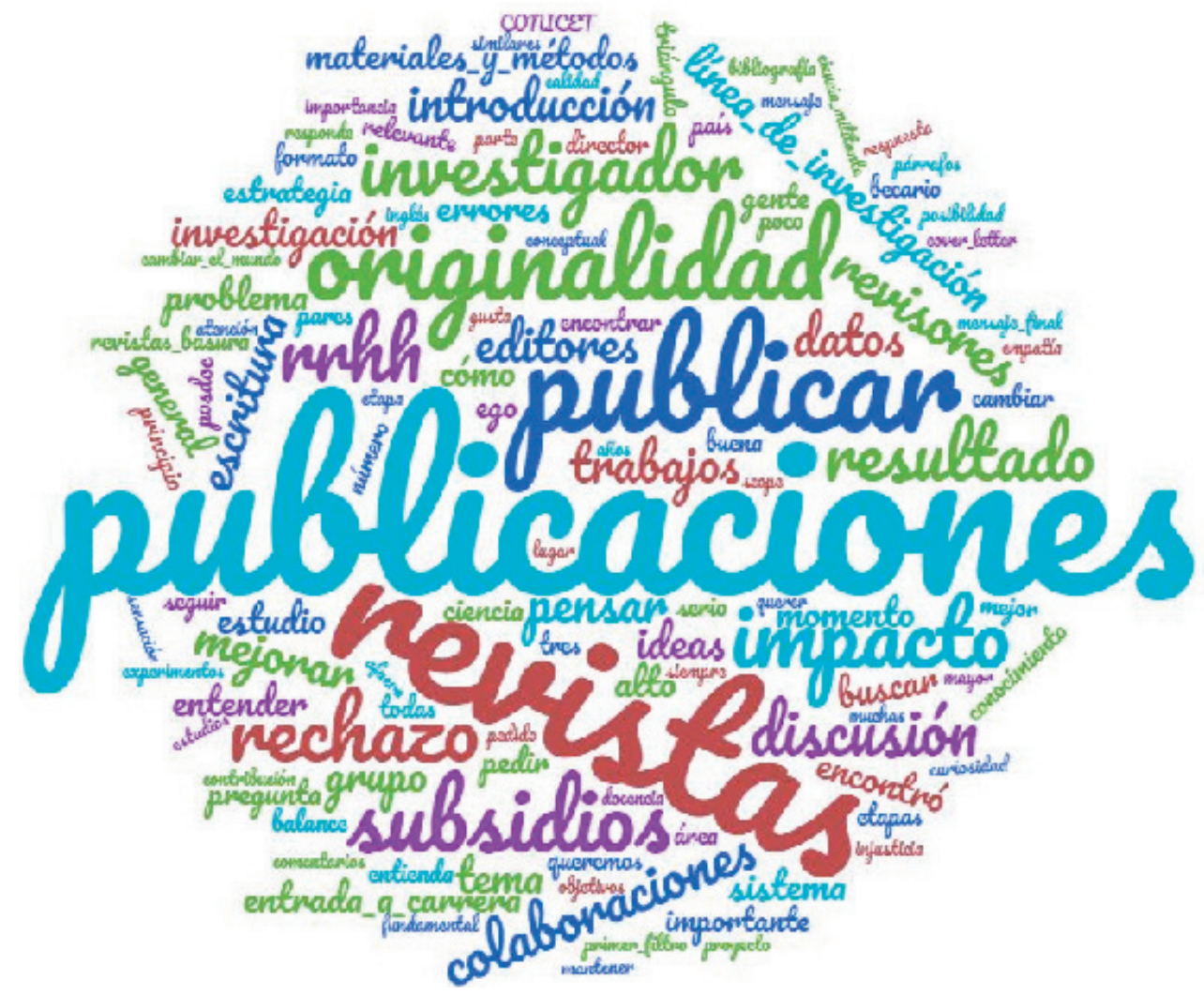

Figura 1. Nube de las palabras que surgieron a lo largo de las reuniones con los ocho investigadores. El tamaño de las palabras representa su frecuencia de aparición en las reuniones; a mayor tamaño, mayor frecuencia (Zygomatic 2016). Los colores son ilustrativos.

Figure 1. Word cloud that emerged in the meetings with the eight researchers. Word size represents their frequency of occurrence during the meetings; the bigger, the more frequent (Zygomatic 2016). Colors are illustrative. 
que consideraban más relevantes durante la etapa posdoctoral. A partir de los apuntes que tomamos en las reuniones armamos una nube con las palabras que surgieron con mayor frecuencia (Figura 1). Esta nube puso en evidencia que el principal aspecto que nos preocupa es cómo lograr publicar nuestros trabajos en revistas científicas de alto impacto. Las palabras más mencionadas fueron 'publicaciones' (1 de cada 4), 'revista' (1 de cada 8) y 'publicar’ (1 de cada 11). En este mismo sentido, otros aspectos que resaltaron fueron la originalidad y el impacto de nuestras publicaciones, el rechazo de los manuscritos y el perfil de los revisores. También se habló de la obtención de subsidios, de la formación de recursos humanos y de la acción de investigar. Finalmente, destacamos que la figura del investigador, la línea de investigación, los datos y las ideas fueron otros temas menos mencionados, pero relevantes para este debate (Figura 1).

A partir de los tópicos que surgieron en las reuniones con investigadores y nuestro debate posterior, dividimos el artículo en tres certezas: 1) la publicación de nuestro trabajo es fundamental, 2) nuestra vocación como investigadores se enfrenta a la necesidad de definir una línea de investigación propia, original y relevante, en un marco de poca claridad y estabilidad en los requisitos y antecedentes necesarios para acceder a la CIC, y 3) el tiempo es limitado y debemos idear una estrategia para jerarquizar las múltiples actividades de nuestra labor. A la vez, cada certeza desplegó incertidumbres que desarrollaremos también en cada sección. Nos pareció interesante compartir nuestras reflexiones respecto de las habilidades y estrategias que debemos desarrollar los investigadores en formación para lograr carreras científicas exitosas, que nos permitan satisfacer nuestra vocación y nuestro papel como investigadores. La intención es abrir el debate y discutir cuáles serían las estrategias para ingresar a la CIC y para desarrollar una carrera científica exitosa. Además, proponemos una encuesta (goo.gl/forms / k185nQG8ABxjL4bo2) dirigida a becarios doctorales, posdoctorales e investigadores que ya tienen una plaza permanente; confiamos en que nos permitirá conocer un abanico real y más amplio de los perfiles y las opiniones de los investigadores del área de las ciencias biológicas, agrarias y ambientales en nuestro país.

\section{La mayor certeza: tenemos que publicar; la incertidumbre: ¿cómo lograrlo?}

Nuestro objetivo como investigadores es hacer visible nuestro trabajo a toda la comunidad científica para generar un impacto en el conocimiento científico y extenderlo a la sociedad. El proceso de publicación en revistas científicas otorga, por un lado, la validación de nuestro trabajo mediante una rigurosa evaluación por pares, y por el otro, la posibilidad de difusión a la comunidad científica y su extensión al resto de la sociedad una vez que está publicado. La revista a la que accedamos definirá el alcance y la audiencia que tendrá nuestra publicación. Publicar en revistas de alto impacto (i.e., primer cuartil) (SCImago 2007) le brindará a nuestro trabajo una mayor posibilidad de generar un aporte al conocimiento, por la gran cantidad de lectores que tienen en todo el mundo. Sin embargo, el nivel de universalidad y novedad de la pregunta de investigación, la aproximación metodológica, la calidad de los datos obtenidos y nuestra capacidad para plasmarlo en un manuscrito son los aspectos que definirán el tipo de revista a la que podremos acceder. Para lograr una publicación de alto impacto debemos lograr la maduración conceptual suficiente para desligar nuestro trabajo del fenómeno local o de las especies de estudio y destacar la originalidad o universalidad de nuestra pregunta o metodología. La discusión de nuestros resultados debe conectarse claramente al 'bache en el conocimiento' planteado en la introducción y no limitarse a la similitud o la diferencia con los resultados de otros trabajos. Es decir, para lograr una difusión exitosa y destacarse en el mar de las publicaciones que existen, nuestros estudios deben saltar del plano de los datos al de las ideas.

Como investigadores en formación, una pregunta que nos debemos formular es si el tiempo para lograr publicar en una revista de alto impacto es compatible con el que tenemos para lograrlo, según las demandas del sistema científico en el que estamos insertos. Ya que la tasa de rechazo en estas revistas ronda el $80 \%$ (Aarssen et al. 2008), los probables múltiples rechazos nos costarán tiempo y trabajo. El rechazo de nuestro manuscrito puede ser resultado de la suma de diferentes desaciertos, aun cuando se cumplan los aspectos mencionados en el párrafo anterior. Por ejemplo, la escritura del resumen y la carta para el editor son los elementos que 
definen si nuestro manuscrito entrará en revisión; una proporción considerable de los rechazos ocurre en esta instancia (e.g., 25\%) (Grod et al. 2009). Otras posibles causas de rechazo pueden estar relacionadas con la claridad con la que exponemos la novedad de nuestra investigación, desde el formato del texto, las tablas y las figuras hasta la sintaxis y la estructura de los párrafos y oraciones. El conjunto de estos desaciertos puede generar una impresión negativa en los editores y revisores al momento de enfrentarse con nuestro manuscrito. Debemos considerar que los revisores son investigadores con poco tiempo disponible y que el proceso de revisión es una actividad anexa, anónima y voluntaria que compite en tiempo con el resto de sus actividades.

Frente a un eventual rechazo, nuestro primer sentimiento será la frustración. Esta es una de las principales causas de abandono de la actividad por parte de los investigadores en formación (Editorial Nature 2016). Sin embargo, lo más relevante es identificar las causas del rechazo de nuestro manuscrito, y utilizarlo como el primer paso para mejorarlo. Es clave entender que el rechazo es más común de lo que se piensa (Aarssen et al. 2008) y que no es algo personal. Debemos valorar el trabajo de los editores y los revisores, y superar la sensación de frustración. Las revisiones definen cómo seguir, por lo que siempre hay que tomar en cuenta (críticamente) sus comentarios y distinguir si el manuscrito tiene problemas graves o no. Si el editor nos da la oportunidad de volver a enviarlo, debemos asegurarnos de que la mejora sea real y visible. Es importante ser honestos y dedicarle el trabajo y el tiempo necesarios a cada respuesta, ser concretos y específicos en las referencias a los cambios en el manuscrito y sólo reenviarlo cuando lo hayamos logrado. Luego de publicado, debemos sentirnos orgullos, comunicarlo y promocionarlo a la comunidad científica y no científica para que se lo pueda encontrar con facilidad y que sea leído y citado la mayor cantidad de veces posible (Baron 2010).

Ante la certeza de querer ser investigadores, las mayores incertidumbres: ¿cómo definir nuestra línea de investigación? ¿Cómo entrar a la CIC del CONICET?

El éxito como investigadores no debería depender sólo de nuestra habilidad para publicar (muchas publicaciones con un impacto promedio alto) (García Romero 2000), sino también de una buena predisposición al intercambio y al desarrollo de ideas y de proyectos nuevos. Por un lado, un punto fundamental que nos inquieta es buscar y crear una línea de investigación propia que satisfaga nuestro interés por la ciencia y que se alinee con los objetivos del organismo al que respondemos. Reconocer nuestras habilidades profesionales y personales nos ayudará a afrontar ambigüedades y a delinear mejor nuestra posición como investigadores en formación. En este aspecto, la interacción con pares y el desarrollo de proyectos de investigación resultan motivadores esenciales. La participación en congresos, reuniones, intercambios y estadías con otros grupos de investigación en el país y en el exterior (García Romero 2000) son actividades que nos enriquecen y que generan nuevas oportunidades y publicaciones (Nerad and Cerny 1999). Sin embargo, también resultan poco (o inciertamente) valoradas por entidades como el CONICET al momento de postularnos a una beca o a la CIC. En la Argentina y en el mundo se acepta que el número y la calidad de las publicaciones en revistas científicas constituye la métrica más empleada a la hora de evaluar a investigadores en formación (Åkerlind 2005).

Por otro lado, nos preguntamos cuánto pesa el contexto sociopolítico al definir la vocación y las estrategias de los jóvenes que queremos desarrollar una carrera científica. En la actualidad, muchos países redujeron el presupuesto destinado a la ciencia, y hoy, la Argentina es un ejemplodeello(e.g., Rodríguez Mega 2016). La revista Nature (2016) remarcó que, a nivel global, el aumento del número de personas dedicadas a hacer ciencia no fue acompañado de un aumento en la cantidad de fondos destinados a dicha actividad. Este contexto determina una competitividad creciente por recursos para investigar, y evidencia que es todavía más necesario contar con publicaciones en revistas de alto impacto para acceder a una planta permanente (Kahn and Ginther 2017). Un escenario más competitivo puede determinar un cambio en la estrategia de los investigadores en formación. Ante la inestabilidad, transitar varias becas posdoctorales representa una gran preparación (Kahan and Ginther 2017), aunque también puede llevar a abandonar la actividad (Editorial Nature 2016). Desde el 2016, el contexto socio-político en la Argentina determina un escenario más exigente para el ingresoa la CIC por una reducción considerable y abrupta de plazas, becas y subsidios para la 
investigación (TSS 2016). A su vez, existe una mayor facilidad para obtener oportunidades en los llamados 'temas estratégicos' (50\% de las becas y plazas) (CONICET 2018), respecto de las áreas de investigación tradicionales; algo similar ocurrió con las consideradas 'áreas geográficas prioritarias' (30\%) hasta el año 2017. Esto último podría desafiar al rediseño de determinadas líneas de investigación como estrategia para el ingreso a la CIC. Consideramos que tanto definir como priorizar temas estratégicos, así como la valuación de las diferentes actividades que pueden llevar a cabo los científicos (temas aquí no desarrollados) son relevantes para el debate en la comunidad científica.

\section{La incertidumbre universal: ¿cómo administramos el tiempo?}

Quienes elegimos seguir la carrera científica debemos tener en cuenta que, a medida que avanzamos, los desafíos cambian y se tornan más complejos. En general, una vez que nos doctoramos puede aumentar de forma considerable nuestra dedicación a la publicación de trabajos científicos, la docencia y la formación de recursos humanos, así como la búsqueda de subsidios, la divulgación científica y la extensión, entre otras actividades. Crear buenas investigaciones científicas se debe basar en un proceso madurativo guiado por la paciencia, ya que es un proceso muy demandante de tiempo (Loehle 2009; FarjiBrener and Ruggiero 2010). Se ha discutido que, aunque el sistema científico argentino aparentemente estimula este proceso, en numerosas ocasiones premia la 'impulsividad' (Farji-Brener and Ruggiero 2010). Por ejemplo, al mismo tiempo que estimula realizar tesis doctorales en un máximo de cinco años, exige al doctorando al menos un trabajo publicado en revistas científicas para obtener una beca posdoctoral, y varias publicaciones para ingresar a la CIC. Estos requisitos a su vez resultan poco claros y dependientes del contexto.

Las reuniones con investigadores formados nos permitieron reconocer que nuestra mayor incertidumbre es cómo lograr la cantidad y calidad de publicaciones necesarias para acceder a la CIC, luego de finalizar una beca postdoctoral (Figura 1). Mientras las becas doctorales tienen una duración de cinco años, las posdoctorales, en general, duran dos. El CONICET sólo permite tomar una beca posdoctoral, aunque existen otras entidades nacionales que también las brindan (incluso, en el área de ciencias agrarias y ambientales, las opciones son mayores que en otras áreas del conocimiento). Postularse a estas becas no es tan frecuente como en otros países, donde el acceso a una planta permanente luego de doctorarse puede demandar varios años y representa una incertidumbre aun mayor que en nuestro país (Åkerlind 2005; Nerard and Cerny 1999). Si tenemos en cuenta que el tiempo es finito, resulta incierto cuál debe ser el balance entre las actividades más valoradas al momento de postularnos a becas o al ingreso a la CIC (i.e., publicación de trabajos científicos), respecto a otras actividades que completan nuestra actividad científica y enriquecen nuestro desarrollo y vocación como investigadores, pero cuyo peso en las evaluaciones es poco claro. Ciertamente, la estrategia a seguir debería estar presente desde el desarrollo del doctorado.

\section{Consideraciones finales}

Luego de las reuniones con investigadores formados y del esfuerzo por sintetizar las ideas que discutimos, identificamos las certezas e incertidumbres más relevantes de la etapa posdoctoral. También detectamos algunas herramientas que nos permitirán desarrollar buenos antecedentes científicos. Durante la etapa posdoctoral se define nuestra vocación científica y también se pone a prueba por los aspectos personales (e.g., lidiar con la frustración de que nos rechacen manuscritos) y por el contexto sociopolítico (cada vez menos becas e ingresos a la CIC). En esta etapa también se encamina nuestra línea de investigación y debemos consolidar nuestros antecedentes para poder postularnos a una posición permanente; todo esto en un tiempo acotado. Para alcanzar nuestro objetivo de ingresar a la CIC debemos planificar una estrategia, cuyos pilares son publicar nuestros trabajos en revistas de alto impacto y elaborar proyectos con ideas originales que representen un avance en el conocimiento. Por un lado, proponemos la necesidad de dar relevancia a nuestros resultados, destacar su originalidad y priorizar el desarrollo de las ideas por encima de la recopilación de datos y de estudios de sistemas locales. Planteamos las incertidumbres respecto a la demanda creciente de antecedentes en el sistema científico argentino y a la poca claridad de los requisitos, aspectos que representan una gran limitante para la continuidad de nuestra carrera. Por otro lado, entendimos que uno de los desafíos más importantes es 
el de administrar el tiempo, considerando que debemos, idealmente durante el doctorado, priorizar el desarrollo de las actividades que nos sumen más antecedentes para poder persistir en el sistema y desarrollar una carrera científica exitosa.

Al escribir este artículo, sentimos la necesidad de ampliar el debate y abrirnos a la diversidad de opiniones de los investigadores en formación y formados en nuestro país. Los invitamos a participar y a difundir una encuesta que diseñamos con el objetivo de evaluar cómo los jóvenes investigadores deberíamos considerar las publicaciones, la vocación científica, el contexto sociopolítico y la administración del tiempo (goo.gl/ forms/k185nQG8ABxjL4bo2, abierta hasta el 31 de marzo de 2019). Esperamos que los resultados de esta encuesta nos permitan hacer una recopilación amplia de experiencias más generales donde cada becario podrá tomar las sugerencias que le sirvan, como así también detectar los perfiles exitosos a los que deberíamos apuntar para hacer ciencia en nuestro país, y así disminuir nuestras incertidumbres.

Agradecimientos. Agradecemos a los investigadores que estuvieron muy bien predispuestos a compartir sus experiencias, pensamientos e ideas: A. Austin, G. Piñeiro, E. Chaneton, M. Aguiar, J. Casal, A. Hall, L. Yahdjian y E. Jobbágy. A G. Piñeiro por su revisión en la primera versión del manuscrito, a los dos revisores anónimos y a E. G. Jobbágy por sus valiosos aportes. A IFEVA por brindarnos el lugar de las reuniones. A $\mathrm{M}$. Druille, J.I. Cagnola y L.I. Pérez que formaron parte de las reuniones y de muchas de las discusiones que dieron lugar a este trabajo. A Antonio, Thiago, Alejo y Felipe que desde su gestación acompañaron el desarrollo de este debate.

\section{REFERENCIAS}

Aarssen, L. W., T. Tregenza, A. E. Budden, C. J. Lortie, J. Koricheva, and R. Leimu. 2008. Bang for Your Buck: Rejection Rates and Impact Factors in Ecological Journals. The Open Ecology Journal 1:14-19. DOI: 10.2174/1874213000801010014.

Åkerlind, G. S. 2005. Postdoctoral researchers: roles, functions and career prospects. Higher Education Research and Development. DOI: 10.1080/0729436052000318550.

CONICET. 2018. Ingresos CIC - Temas Estratégicos y Tecnología. URL: convocatorias.conicet.gov.ar/temasestrategicos.

Baron, N. 2010. A guide to making your science matter. Escape from the ivory tower. Island Press, Washington DC, EE.UU.

Editorial Nature. 2016. Early-career researchers need fewer burdens and more support. Nature 538:427-427. DOI: 10.1038/538427a.

Farji-Brener, A. G., and A. Ruggiero. 2010. ¿Impulsividad o paciencia? Qué estimula y qué selecciona el sistema científico argentino. Ecología Austral 20:307-314.

García-Romero, A. 2000. El efecto de la estancia postdoctoral en la productividad científica. URL: URL: goo.gl/ $1 \mathrm{MA} 1 \mathrm{Nb}$.

Grod, O. N., C. J. Lortie, and A. E. Budden. 2010. Behind the shroud: a survey of editors in ecology and evolution. Frontiers in Ecology and the Environment 8:187-192. DOI: 10.1890/090048.

Kahn, S., and D. K. Ginther. 2017. The impact of postdoctoral training on early careers in biomedicine. Nature Biotechnology 35:90-94.

Loehle, C. 2009. Becoming a Successful Scientist: Strategic Thinking for Scientific Discovery. Cambridge University Press, Cambridge, New York.

Nerad, M., and J. Cerny. 1999. Postdoctoral Patterns, Career Advancement, and Problems. Science 285, 1533. DOI: 10.1126/science.285.5433.1533.

Rodríguez Mega, E. 2016. Argentina's scientists engulfed in budget crisis. Science. DOI: 10.1126/science.aal0388.

SCImago. 2007. SJR - SCImago Journal and Country Rank. Retrieved July 21, 2015, from URL: www.scimagojr.com.

Vicens, Q., P. E. Bourne, and K. Karbstein. 2009. Ten Simple Rules To Combine Teaching and Research. PLoS Computational Biology 5:e1000358. DOI: 10.1371/journal.pcbi.1000358.

TSS. 2016. Agencia de Noticias Tecnológicas y Científicas de la Universidad Nacional de San Martín. URL: www.unsam.edu.ar/tss/la-ciencia-recortada.

Zygomatic. 2016. Generador de nube de palabras y creador de nubes de etiquetas gratis y online. URL: www.nubedepalabras.es. 